\title{
Comparitive Analysis Of Intramedullary Versus Extramedullary Fixation In Subtrochanteric Fractures
}

\author{
Poluboina Aswin Kumar ${ }^{1}$, Ganesan Ganesan Ram ${ }^{2}$, \\ Giriraj Harshavardhan ${ }^{3}$, Thamodaran Balasugumar ${ }^{4}$, Kunal Pate ${ }^{5}$, \\ Phagal Varthi Vijayaraghavan ${ }^{6}$ \\ 1,2,3,4,5,6, (Orthopedics / SRMC-Sri Ramachandra University, India)
}

\begin{abstract}
Prospective study of 40 cases of subtrochanteric fracture treated either extramedullary fixation intramedullary fixation in Sri Ramachandra medical center April 2011to June 2013. Inclusion criteria are all traumatic subtrochanteric fractures, exclution criterias are all open fractures paediatric fractures, pathologic fractures. We had 20 extramedullary and 20 intramedullary fixations we used schatzker and Lambert scoring system, mean follow up for 2 years. The most important factor to prevent the failure of fixation may be due to inadequate initial reduction. The most important factor to prevent the failure of fixation is anatomical reduction of fracture and restoration of medial buttress. In our comparative analysis of intramedullary versus extramedullary fixation of subtrochanteric fractures. Intramedullary devices give superior results than extramedullary devices. But the most important factor to prevent the failure of fixation is Anatomical reduction of fracture at time of surgery.
\end{abstract}

Keywords: Anatomical reduction, Extramedullary, Intramedullary, Subtrocahnteric femur fractures, schatzker and Lambert scoring system,

\section{Introduction}

Injury to the femur, the largest bone in the body, presents a challenging situation to the orthopedic surgeons. The subtrochanteric fractures has long been recognized as the most difficult of these injuries to treat and the reported mortality ranges from $8.3 \%$ to $20.9 \%$. Subtrochanteric fracture treated with intramedullary or extramedullary fixation. Currently many implants are used to treat these fractures. Extramedullary fixation needs big skin incision, extensive soft tissue dissection and more blood loss. Biomechanically lateral plate devices allow the fracture to collapse into varus if the medial buttress is not automatically restored. These can lead to delayed union, nonunion, and subsequently implant failures. Intramedullary fixation needs smaller incision, closed procedure, minimal blood loss. Stable fixation. An ideal implants for fixation of subtrochanteric fractures is still evolving, we thought it would be apt to compare and analyse the functional and radiological outcome of subtrochanteric fracture treated by extramedullary fixation as well as the intramedullary fixation.

II. Aim

To compare and analyse intramedullary versus extramedullary fixation in subtrochanteric fractures.

\section{Materials And Methods}

Prospective study consists of 40 subtrochanteric fractures treated in SRMC from the period of April 2011-June 2013. In these Inclusion criteria are all traumatic subtrochanteric fractures, exclution criterias are all open fractures paediatric fractures, pathologic fractures. In study we had 20 patients in extramedullary fixation and 20 patients in intramedullary fixation. We had $65 \%$ of male patients and $35 \%$ of female patients. In our study patients age group between 40-60 years and majority of patients mode of injury bis due to road traffic accidents. There study was assesed by schatzker and Lambert scoring system. All patients are followed up at regular intervals, 6 weeks, 3 months, 6 months, 12 months, 24 months.

\section{Results}

In our study intramedullary fixation group excellent and good results accounted to $75 \%$, fair results accounted to $20 \%$, poor results accounted to $5 \%$.

In extramedullary fixation group excellent and good results accounted to 55\%, fair results accounted to 
$35 \%$, poor results accounted to $10 \%$.

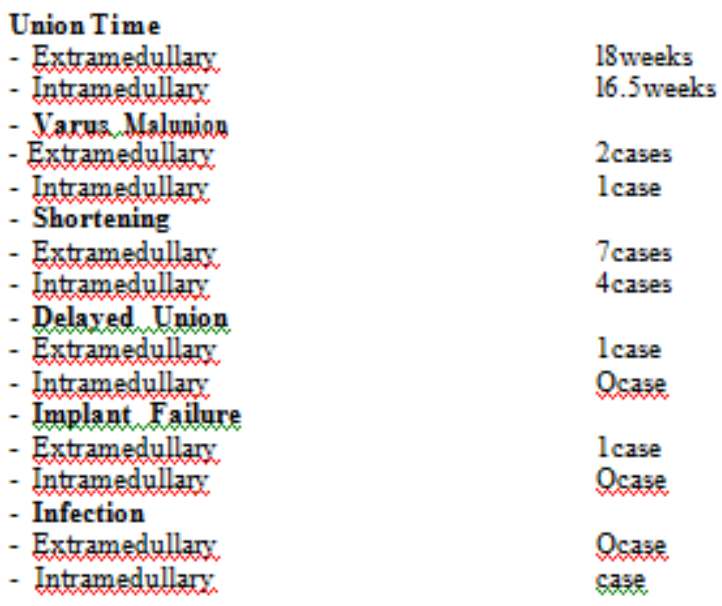

\section{Discussion:}

Management of subtrochanteric fractures of the femur poses a great challenge to orthopaedic surgeon. In adults these fractures are usually the result of high energy trauma and are often comminuted at medial cortex. In the need for early mobilisation and osteoporosis makes the selection of implant are important issue. Many clinical and biomechanical studies have analysed the results of different implants. Treatment of subtrochanteric fractures of the proximal femur is still associated with some failures, the reasons being; disregard for biomechanics, over estimation of the potentials of new surgical techniques or new implants and poor adherence to establish procedures. High stress concentration that is subject to multiple deforming forces, slow healing time because of predominance of cortical bone, decreased vascularity, higher incidence of complications reported after surgical treatment compels the surgeon to give a second thought regarding selection of proper implant. The goal of surgical treatment of subtrochanteric fractures are

1. Stable reduction

2. Stable internal fixation

3. Early mobilization

4. Early functional rehabilitation of the limb

The most common current modes of fixation are

Intramedullary fixation and

Extramedullary fixation.

Intramedullary fixation allows the surgeon to minimise soft tissue dissection there by reducing surgical trauma, blood loss, infection and wound complications.

Extrmedullary fixation is associated with blood loss due to extensive dissection, while chances of implant failure and delayed union or non union are more.

Our study consists of 40 adult patients with subtrochanteric fracture of femur, who were randomly treated with intramedullary fixation and extramedullary fixation.

Fractures were classified according to seinsheimer's classification and the results was assessed by schatzker and Lambert scoring system and found that there is statistically significant. Intramedullary fixation superior than extramedullary fixation. According to schatzker and Lambert scoring system in extramedullary fixation there is Excellent-2, Good-8, Fair-8, poor-2. In intramedullary fixation there is Excellent-4, Good-II, Fair-4, poor-I. 


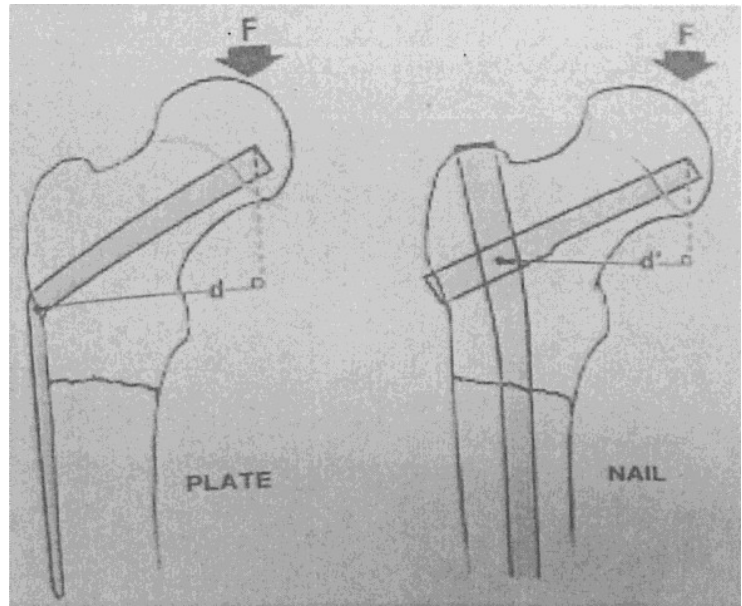

Intramedullary nails are biomechanically superior because of the reduce the distance to the tip of the portion of the implant in the femoral head than the plate systems that are on the lateral border of the femur, the shorter the distance over the which the bending force act, the lower the moment of the implant. This is important in the fracture were the medial butters cannot be restored. In this study the percentage of failure in fixation category is more in the extramedullary than the intramedullary. These failure of fixation may be due to inadequate initial reduction. The most important factor to prevent the failure of fixation is Anatomical reduction of fracture and restoration of medial buttress. If we achieve initial reduction we can maintain neck shaft angle which plays a key role.

We have two cases of poor results in extramedullary fixation and 1 case of poor result in intramedullary fixation.

Case 1 Subtrochanteric fracture type 5 patient had underwent dynamic condylar screw fixation. Patient immediate post-operative neck shaft angle is 98 degrees, the fracture continued to heal in varus alignment. The fracture fragment's were not reduced properly and neck shaft angle was not restored. Hence this fracture went for varus non-union of proximal femur.

Case 2 The patient had poor results initially due to inadequate fixation following the subtrochanteric fracture within two months implant failure occured, screws brokeout and plate had lifted out from the bone, this was revised using longer DHS with adequate screws. This fracture went it for union maintaining neck shaft angle. So this was consider poor result initially.

Case 3 This patient had poor result as the PFN fixation was done without proper reduction of fracture fragment and hence neck shaft angle was not maintained. The fracture went for varus malunion.

\section{Scoring Result}

In these functional and radiological outcome was assessed by Schatzker and Lambert scoring system. In our study intramedullary fixation group excellent and good results accounted to $75 \%$, fair results accounted to $20 \%$, poor results accounted to $5 \%$.In extramedullary fixation group excellent and good results accounted to $55 \%$, fair results accounted to $35 \%$, poor results accounted to $10 \%$.

\section{Conclusion}

1. Subtrochanteric fractures are common in high velocity trauma

2. High stress concentration, slow healing time predominance of cortical bone and difficulty in getting biomechanically sound reduction because of usual medial comminution has led to evolution of various fixation devices.

3. In our comparative analysis of intramedullary fixation vs Extramedullary fixation of subtrochanteric fractures.

a) Intramedullary device gives superior results when compare to extramedullary device.

b) But the most important factor to prevent the failure of fixation is Anatomical reduction of fracture at time of surgery.

c) Maintaining neck shaft angle and anatomical reduction is key to success of fixation.

d) If we do not maintain neck shaft angle intramedullary fixation goes into mal union, extramedullary fixation goes into non union. 


\section{References}

[1]. Paul R.T. Kuzyk, MASc, MD, FRCS(C), Mohit Bhandari, MSc, MD, FRCS(C), Michel D. McKee, MD, FRCS(C), Thomas A. Russell, MD and Emil H. Schemitsch, MD, FRCS(C). Intramedullary versus extramedullary fixation for subtrochantric femur fractures.

[2]. Frederick J Kummer, Ola Olsson, Charles A Pearlman, Leif ceder, Sune Larsson and Kenneth J Koval. Intramedullary versus extramedullary fixation of subtrochantric fractures A biomechanical study.

[3]. Allis OH: Fractures in the upper third of femur exclusive of the neck. Med. News 59: 585-589:1891.

[4]. Asher MA, Tipper IW: Compression fixation of subtrochanteric fractures Orthop 117: 202. 208:1976.

[5]. Asheesh Bedi, MD, T.Toan Le MD: Subtrochanteric femur fractures. Orthop Clin North Am 35 :

[6]. $473-483 ; 2004$

[7]. Bose WI: A preliminary experience with Russell - Taylor reconstruction nail femoral for complex fractures. J Trauma 32:71-76; 1992.

[8]. Boyd HB, Griffin LL: Classification and treatment of trochanteric fractures. Arch Surg 58: 853-86u), 1949. Campbell's Operative Orthopaedics Vol. 3: Edited by Terry Canale; $10^{\text {th }}$ edition.

[9]. Cech H, Sonsna A: Principles of surgical treatment of subtrochanteric fractures. Orhop Clin North Am 5: 651-662:1974.

[10]. Charnley G J, Ward A I: Reconstruction femoral nailing for non- union of Subtrochanteric fracture: a revision technique following dynamic condylar screw failure. In! orthop: 20(1\}: 55 - 57; 1996.

[11]. Clawson D.I ( Trochanteric fractures treated by sliding screw platefixation Journal of Trauma 4:337; 1964

[12]. Cochran GVR: Implantation of strain gauges in hone in VIVO. Biomechanics 119;1972.

[13]. Ender SW: Treatment of pcrtrochantcric and subtrochunteric traclurcs of femur with 'Enders pins. Acta Chir Austriaca 1: 40-42; 1970 .

[14]. Feilding JW: Subtrochanteric fracture. Clin Orihop 92:86;89, May 1973.

[15]. Comparitive analysis of intramedullary versus extramedullary fixation in subtrochanteric fractures

[16]. Feilding JW, Cochran OVB, Zickel Rc: Biomechanical characteristics and surgical management of Subtrochameric fractures. Orthop North 1lm5: 629-fJ50; 1974.

[17]. Frankel V, Burstein : Orthopaedic biomechanics. Lea and Fcbigcr Philadelphia Lea\& 1-cbigcr;1970

[18]. Frederick J Kummer: Intramcduttary versus extramedullary fixation of Subtrochanteric fractures. Acta Orthop Scand j998:69(6j: 580584.

[19]. French BG. Use of an interlocked cephalomedullary nail for subtrochanteric Iracturc stabilizariou. Clin Orthop 348: 95 - 100: 19,98.

[20]. Gibbons CL: Use of the Russell. Taylor reconstruction nail in femoral shaft fractures. Injury 26:389 - 392: 1995. 